\title{
Intervención Psicoeducativa en el Aula con TDAH
}

\section{Psychoeducation Management in the Attention-deficit Hyperactivity Disorder (ADHD) Classroom}

\author{
José Luis Galve Manzano \\ Colectivo para la Investigación y Desarrollos Educativos Aplicados
}

\begin{abstract}
Resumen. Trataremos de mantener un equilibrio entre lo que se tiene que hacer, lo que se suele hacer, y lo que se puede hacer. En TDAH, como alumnado con necesidad específica de apoyo educativo procede la determinación de posibles necesidades educativas, así como la adopción de medidas extraordinarias, si así procediese. Fases de la atención al alumnado: a) detección de dificultades; b) traducción de dichas dificultades en términos de necesidades educativas; y c) diseño de la respuesta educativa en función de dichas necesidades. Esta problemática se ha venido abordando desde diferentes enfoques de intervención (médico, psicoeducativo o psicopedagógico, y “combinado”). Deben recibir atención tanto dentro como fuera del aula (enfoque multidisciplinar) con intervenciones desde las perspectivas médica, psicológica y psicopedagógica; beneficiándose con el empleo de las técnicas de los enfoques conductuales y cognitivos. Debe tenerse en cuenta el estilo de enseñanza-aprendizaje. Cobra especial importancia la formación de docentes, y de padres, así como la intervención directa con el alumnado. Desde el punto de vista de la intervención, hay que abordar los ámbitos: comportamental, de los aprendizajes, emocional y social.

Palabras clave: TDAH, atención a la diversidad, enfoques de intervención, perspectivas de intervención, ámbitos de intervención TDAH.
\end{abstract}

\begin{abstract}
We will try to keep a balance between what should be done, what is usually done, and what can be done. If we consider ADHD students as having specific support needs, we can determine possible educational needs and take extraordinary measures if necessary. These needs will be attended to by taking three different steps: a) detection of difficulties; b) expression of such a difficulty in terms of educational needs c) design of the educational response in terms of these needs. This problem has been addressed from the different points of view about intervention (medical, psychoeducational or psychopedagogical, and "combined", "interactionist" or eclectic). It should receive attention both within and outside the classroom (multidisciplinary approach) with interventions from the following perspectives: medical, psychological and psychopedagogical. Thus, this problem will benefit from the use of cognitive behavioral approaches. The style of teaching and learning should always be taken into account. Training is particularly important for teachers and parents, as well as direct intervention with the students. From the point of view of intervention, we must address the following areas: behavioral, of learning, emotional and social.
\end{abstract}

Key words: ADHD, attention to diversity, approaches to intervention, perspectives of intervention, areas of ADHD intervention.

La correspondencia sobre este artículo debe enviarse a los E-mail:

jlgalve@telefonica.net; cideas@telefonica.net 


\section{Introducción}

Sin duda es uno de los muchos temas que preocupan en el mundo educativo, pero no deja de ser uno más de las muchas demandas de intervención que tienen los profesionales de la educación (profesores, orientadores, etc), no siendo una demanda nueva, pues desde los orígenes de la escuela han estado los alumnos con este perfil. Han estado sin tipificar, o diagnosticar al menos como en la actualidad.

Cuando me propusieron este trabajo, la primera duda que me surgió fue: ¿cuento lo que ya viene en los manuales y en la mayoría de las experiencias publicadas?, o ¿cuento lo que realmente pasa en el ámbito educativo con los alumnos con TDAH?. Después del bombardeo bibliográfico y formativo sobre el tema, cabe preguntarse: ¿qué puede ser de interés para los participantes en este tipo de jornadas?. Considerados los TDAH como alumnos con necesidades educativas, o mejor dicho, "alumnado con necesidad específica de apoyo educativo", cabe preguntarse: ¿se les atiende de forma diferenciada de los demás alumnos, y sobre todo, con respecto a los demás alumnos con necesidades específicas (acnees), ¿los TDAH, son algo nuevo o siempre han existido en la escuela?. Desde esta postura vamos a tratar de mantener un equilibrio entre lo que se tiene que hacer, lo que se suele hacer, y lo que se puede hacer.

En la mayoría de los manuales, monografías y estudios publicados sobre el tema existe bastante coincidencia en lo que se tiene que hacer en el ámbito educativo, pero rara vez se consideran o se tienen en cuenta los contextos en los que se realiza la labor docente en estos centros, y que sin duda mediatiza la intervención, ya que una cosa es la teoría y otra muy distinta las circunstancias en las que se desarrolla la práctica educativa cotidiana.

En general, los estudios experimentales o de investigación en este campo adolecen de las posibilidades de generalización de ese modelo de intervención, ya que se hacen en un contexto que podríamos denominar de "laboratorio", donde todas las partes (profesores, padres, alumnos,..) están dispuestas a implicarse y se dan las circunstancias para desarrollar esa actividad, más bien como un estudio o trabajo piloto "controlado".

Por otro lado, es uno de los "temas monográficos" de la última década, y nos tememos que pueda pasar como en la década de los ochenta con la dislexia, o en los noventa con las habilidades sociales y la mejora de la inteligencia; donde estos temas fueron "quemados" fundamentalmente por dos motivos. El primero por la falta de formación de los agentes implicados en dar solución a esta temática y, en segundo lugar, por las dificultades contextuales, ya que existía y existe una gran dificultad horaria para abordar esta problemática en el contexto horario disponible en un centro educativo. Igual que pasa con otras muchas temáticas de este estilo, que requieren intervención clínica y pedagógica simultánea y coordinada.

Pero además, la experiencia nos dicta que el alumnado con déficits en la capacidad atencional y de autocontrol, no tiene por qué ser necesariamente TDAH, y que no necesariamente a unos y a otros, les implicarán deterioro en su vida académica, social o familiar. Por tanto, no todos podrán ser diagnosticados como TDAH, si no que algunos serán alumnos/as con problemas o dificultades en las áreas de atención y autocontrol; del mismo modo, estos alumnos -como cualquier otro alumno/a- pueden beneficiarse de las mismas estrategias cognitivoconductuales que se utilizan en los alumnos con TDAH. Dicho de otra forma, el hecho de ser un tdah no necesariamente predice un fracaso escolar, aunque suelen tener una evolución adaptativa costosa en todos los aspectos.

Centrándonos en la intervención desde el ámbito educativo, procedería conocer cómo se organiza la atención a la diversidad desde la acción tutorial. Según nuestro modo de ver, la función tutorial, en lo que se refiere a la atención a la diversidad, consiste en un proceso, en el que básicamente, se empieza a actuar cuando se detecta "algún mal funcionamiento" de un alumno/a concreto. Lo primero es lo que puede ser denominado como de detección y consulta. Esto es útil para conocer de primera 
mano, a través del tutor/a o a través del profesorado que con él comparte la responsabilidad de la atención de un grupo de alumnos, la existencia de uno que presenta características diferenciales, que hacen difícil que rinda satisfactoriamente en la escuela. En una primera fase, se empieza a analizar las características de ese alumnado concreto (reuniendo información, consultando a los otros profesores, a la familia, o incluso recibiendo información de los servicios externos a través de los padres,...) con la intención de plantear alguna hipótesis que explique la razón de las dificultades detectadas. Esta fase podría esquematizarse de la siguiente forma (Trallero, 2008):

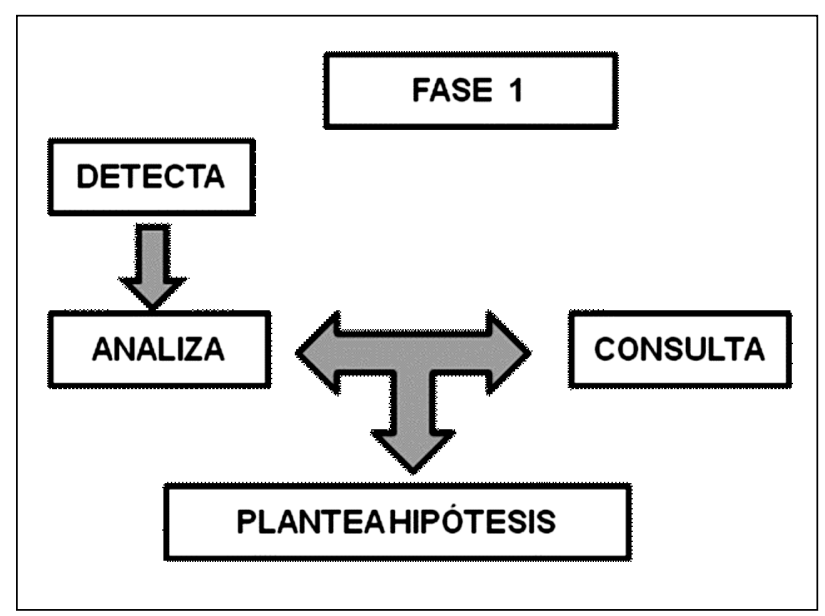

Tras esto comienza una segunda fase, es la fase de constatación de necesidades educativas y toma de decisión, en la que el tutor/a y el equipo de profesores del curso en cuestión deben adoptar medidas de carácter ordinario (que afectan fundamentalmente a los principios pedagógicos, metodológicos y de organización), aquellas que están a su alcance y que comprometen exclusivamente a ellos, pero cuya adopción de nuevo puede ser consultada y discutida con otros especialistas u orientadores.

Si las medidas ordinarias propuestas y llevadas a cabo surten un efecto positivo, si las hipótesis planteadas eran correctas y las dificultades pueden darse por controladas, entonces pueden seguir el mismo proceso de enseñanza-aprendizaje que con el resto de sus compañeros. Pero, si por el contrario, no se ha conseguido el éxito, se debe entrar en la tercera fase, que es la fase de determinación de posibles necesidades educativas especiales y la adopción de medidas extraordinarias, en la que el protagonismo se comparte con el orientador/a, que es el responsable de la evaluación psicopedagógica que llevará o no a la constatación de tales necesidades, con la consiguiente obligación de que sea adaptado el currículo de manera significativa.

Tras tomar conciencia de las dificultades, que a veces entraña a tutores y profesorado en general, el abordar sistemáticamente un gran número de casos de alumnos que por una razón o por otra "no funcionan" con normalidad en el aula, utilizamos desde hace tiempo un instrumento que denominado protocolo de determinación de necesidades educativas (Galve y Trallero, 2003), con la intención de contribuir a la toma de conciencia por el profesorado ordinario de la responsabilidad que comporta la existencia de alumnos con dificultades, y en la adopción de medidas al respecto.

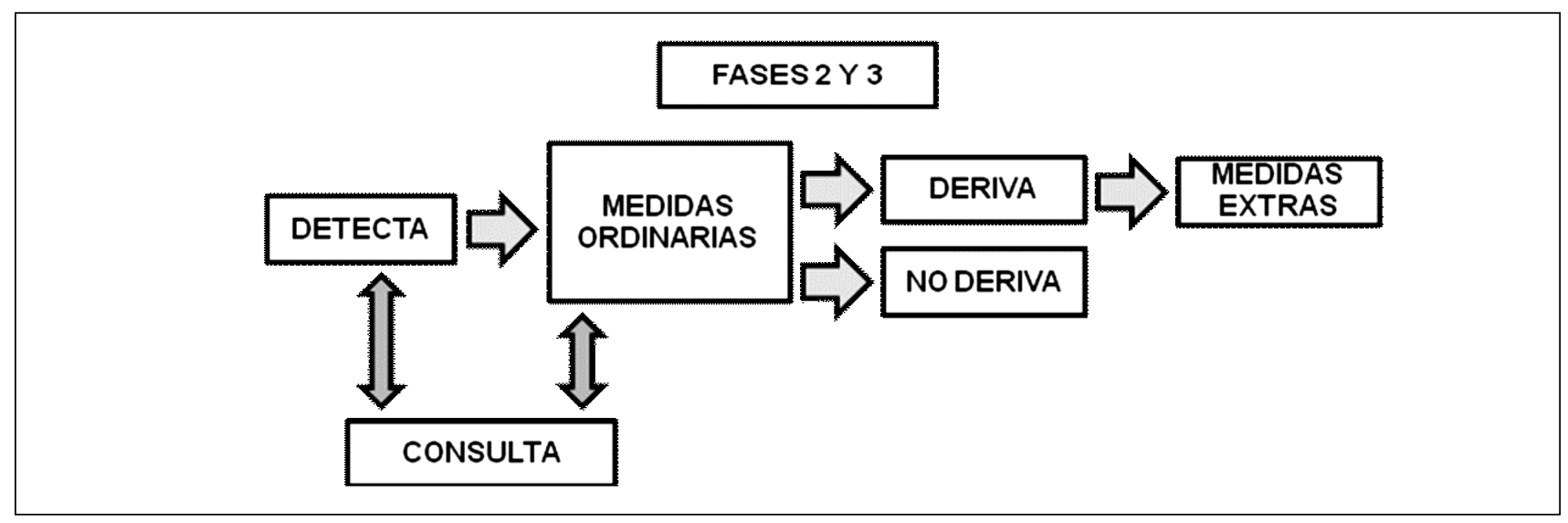


Entendemos que responsabilizarse de un problema y tomar medidas al respecto exige como mínimo lo siguiente:

- Ser capaz de definir el problema.

- Plantearse hipótesis acerca de las causas que lo motivan.

- Plantearse medidas para solucionar el problema partiendo de sus causas.

- Valorar la viabilidad de esas medidas.

- Calibrar las repercusiones positivas y negativas de las mismas.

Este proceso, pretende ser una mera reflexión observacional por parte de los profesores con la finalidad de realizar una aproximación sucesiva a la respuesta educativa necesaria para ese alumnado. Para ello se sugiere seguir el siguiente procedimiento:

$1^{\circ}$ Realizar una primera aproximación al caso: definición lo más explícita y objetiva del problema, del ámbito o ámbitos en los que se produce.

$2^{\circ}$ Formular una primera hipótesis acerca del posible origen del problema a partir de la observación, conocimiento de antecedentes,... sin despreciar factores contextuales, como posibles causas de las dificultades observadas, y/o que pudieran estar en la actualidad agravando o minimizando las dificultades observadas.

$3^{\circ}$ Realizar una primera valoración de las posibilidades de atención en el entorno normalizado, con o sin medidas extras, tras valorar el problema de manera contextualizada. $4^{\circ}$ Analizar las posibles medidas ordinarias que pudieran ser eficaces a nivel de centro y aula, y las repercusiones en la misma, es decir, los beneficios y perjuicios que pudieran ocasionar al resto.

$5^{\circ}$ Reflexionar sobre los resultados de las medidas adoptadas anteriormente, y la coincidencia o no con las que se presumen eficaces.

$6^{\circ}$ Analizar las medidas excepcionales a adoptar, con indicación de los componentes curriculares que podrían verse afectados, lo que viene a ser un "prediseño" de Adaptción Curricular Individual (ACI).

A partir de este momento, entraría en juego la actuación del servicio de orientación; pero teniendo en cuenta que cuando se trata de alumnos con necesidades especiales, se tiende a confundir el carácter de algunas de ellas. Son varias las instituciones que inciden por lo general en la intervención de esta temática; y sobre todo, en los de menor edad, realizando a veces tareas muy parecidas, pero con finalidades diferentes y complementarias. Así por ejemplo, puede darse la paradoja de que las instituciones sanitarias, educativas y sociales podrían estar atendiendo a la vez al mismo alumno/a en sus respectivos servicios sin que, por lo menos en teoría, se solapasen las actuaciones ni mucho menos se coordinen.

Deberíamos considerar al menos en teoría, porque en la práctica resulta francamente difícil delimitar, cuáles de las necesidades presentes son de carácter educativo y cuáles rehabilitador, pero realizar ese

\section{Sintesis del proceso de respuesta a la diversidad}

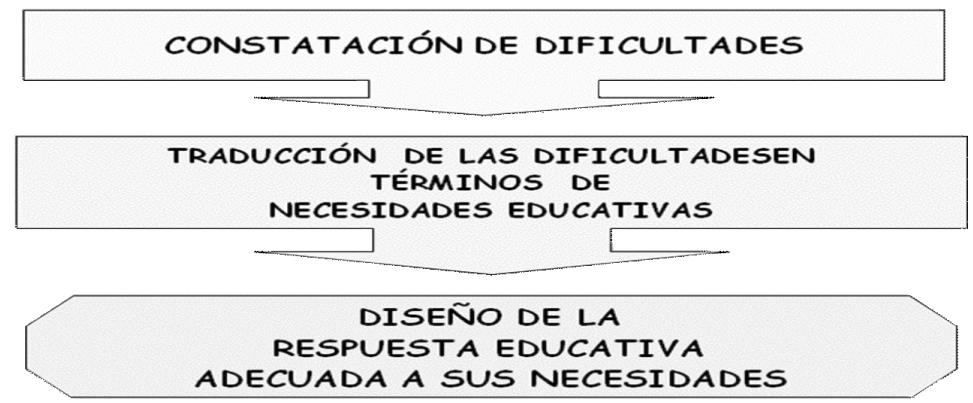


esfuerzo de discernir entre unas y otras es parte del trabajo del orientador, cuya función diagnóstica consiste en dictaminar necesidades estrictamente educativas, especiales o no. O lo que es lo mismo, necesidades que afectan a la adecuada implementación del currículo ordinario, formando parte activa y fundamental del proceso de atención a la diversidad, que resumimos en tres fases: a) detección de dificultades; b) traducción de dichas dificultades en términos de necesidades educativas; y c) diseño de la respuesta educativa en función de dichas necesidades.

El orientador escolar, cuyo perfil profesional, recordemos, puede ser el de psicólogo, pedagogo o psicopedagogo, ni tiene, ni debe, ni puede ejercer como psicólogo clínico, neurólogo o pediatra. Debe limitarse a realizar su función, concluir sus evaluaciones con la determinación de necesidades educativas, que de ser especiales supondrán la propuesta de medidas también especiales.

Partiendo de todo esto veremos con posterioridad cómo la intervención con TDAH requiere normalmente la coordinación con los servicios educativos y de los servicios externos (públicos o privados). Es preciso recordar esto cuando se trata de la temática que nos ocupa, ya que no es cuestión baladí. Si no, intenten responderse a las siguientes preguntas: "¿resulta imprescindible saber si el alumno/a X padece un Trastorno por Déficit de Atención con o sin Hiperactividad para ser atendido adecuadamente en el ámbito escolar?; ¿podríamos medicarle?, y en caso afirmativo, ¿bajo qué pautas o condicionantes?. ¿Hasta qué punto son sus dificultades lectoescritoras o de aprendizaje en general, la causa de su conducta hiperactiva, inatenta, impulsiva,...?, o viceversa. ¿Es hiperactivo porque es inatento? $o$... “

La cuestión es intentar solventar los problemas que, procedentes del ámbito personal, socio-familiar o académico, están interfiriendo en el desarrollo del alumno/a, de ahí que el diseño de un análisis funcional y las propuestas de intervención en la escuela, se puedan ver completadas con otras sugerencias que vayan más allá que el derivar a otros servicios, fundamentalmente sanitarios, en los que apoyarse y a los que prestar apoyo, aunque como ocurre en muchas ocasiones, y ésta es un buen ejemplo, no se suelan atender debidamente por la falta de coordinación entre estos servicios.

El informe, por supuesto, debe incluir propuestas de intervención, y en este caso lo debe hacer de una manera amplia. En la práctica cotidiana, estas propuestas en los informes psicopedagógicos suelen ser meras pinceladas, no más que sugerencias que puedan servir de guía para la elaboración de la adaptación curricular o un programa de intervención individual posterior, que será competencia del equipo docente que atiende al alumno/a en concreto, y coordinados por el tutor/a. Pero se han preguntado alguna vez: ¿por qué ocurre esto?, o sea, ¿a qué es debido que la intervención desde lo indicado en el informe sea "una mera enumeración", más que una propuesta de intervención?.

\section{Breve conceptualización}

En este apartado no pretendemos hacer una conceptualización, ni siquiera una descripción de la sintomatología o las características de TDAH, ya que no es el objetivo de este trabajo. Sin embargo si vemos necesario hacer una breve enumeración para guiar después la propuesta de intervención pedagógica, situando este trabajo en un contexto más global.

Por lo general, se admite que el Trastorno por Déficit de Atención con o sin Hiperactividad (TDAH) es el término que se utiliza en la actualidad para describir una situación, temporal aunque habitualmente crónica, de inadaptación o desajuste al medio social, a causa de la interacción de una característica congénita, denominado Déficit de Atención Sostenida e Hiperactividad, con los sistemas de valores, actitudes y hábitos de comportamiento de los restantes miembros de la familia, escuela o sociedad en general. El trastorno por déficit de atención con o sin hiperactividad, es un trastorno que se inicia en la infancia y se caracteriza por dificultades para mantener la atención, hipe- 
ractividad o exceso de movimiento e impulsividad o dificultades en el control de los impulsos.

Las características principales del alumnado con TDAH son las siguientes:

a) Déficit de atención: presentando escasa atención sostenida, o persistencia en la realización de tareas. Dicho de otra forma, dificultades para mantener la atención durante períodos de tiempo razonables.

b) Hiperactividad-hiperkinesia: suelen presentar una excesiva movilidad, inquietud constante, infatigables, y aparentemente innecesaria para lograr los fines que desean. Esto afecta en gran medida a sus aprendizajes, sobre todo los que implican un componente cognitivo por el déficit en atención sostenida. Con frecuencia también muestran impulsividad.

c) Retrasos en habilidades cognitivas: Suelen carecer de habilidades en estrategias de solución de problemas, manifestando mucha menos fluidez y flexibilidad cognitiva, tratando de resolver la situación o tarea de forma rápida y poco reflexiva. Con frecuencia, muestran una memoria a corto plazo disminuida, afectando frecuentemente a la comprensión de instrucciones y a su ejecución.

d) Deficiencia en el control de las emociones: suelen mostrase como alumnos menos "maduros", infantiles, rápidamente alterables, y fácilmente frustrables por los acontecimientos.

e) Torpeza motriz: suelen mostrar dificultades en motricidad fina.

f) Memorización: suelen presentar dificultades en tareas que impliquen memoria de trabajo y memoria a largo plazo.

g) Variabilidad o inconsistencia temporal: aparecen fluctuaciones constantes en su rendimiento, yendo desde completar sus tareas de una manera rápida y correctamente, a realizarla de una manera pobre, con poca precisión, y con relativa incorrección.

h) Problemas de rendimiento académico: con frecuencia aparecen retrasos y fracasos escola- res, ya que las carencias anteriores conllevan aprendizajes incompletos y/o incorrectos de habilidades básicas para un adecuado rendimiento académico.

i) Problemas de adaptación social: fundamentalmente por el déficit en la adquisición de habilidades cognitivas que les permitan regular su comportamiento.

j) Déficits de autoconcepto, autoestima y asertividad: como consecuencia de la acumulación crónica de frustraciones y castigos, que suelen ir en su mayor parte dirigidos a su persona, más que a su comportamiento inadecuado, debido al modelo socio-educativo de nuestra cultura.

k) Problemas emocionales: con frecuencia, suelen mostrar indicadores de ansiedad y estrés, junto a escasa asertividad y bajo autoconcepto y autoestima.

Esto, en gran medida, suele estar generado o potenciado por las demandas o presiones de su contexto socio-familiar y/o educativo.

A modo de síntesis, podríamos decir que esta sintomatología suele aparecer desde el inicio de su escolaridad, con cambios atencionales frecuentes y excesiva actividad motora, continuando estos indicadores de forma evolutiva, teniendo una consistencia temporal a lo largo de su escolaridad, sobretodo si no se ha hecho una intervención específica. Aunque también hay que tener en cuenta que estas manifestaciones conductuales se atenúan con la edad, sobre todo a partir de la adolescencia.

\section{Enfoques para la intervención}

En los últimos tiempos esta problemática se ha venido abordando desde diferentes enfoques de intervención, con diferentes tratamientos enfocados sobre todo a disminuir la sintomatología.

Tipos de enfoques:

a) Enfoque médico. Básicamente sostiene la existencia de factores neurológicos, genéticos, metabólicos,... como base del trastorno. Inter- 
viniendo fundamentalmente a través de medicación psicoestimulante. Sigue siendo un tema controvertido, pero que al no ser el objeto de este trabajo no vamos a entrar en ello.

b) Enfoque psicoeducativo o psicopedagógico. Sostiene que es un comportamiento aprendido y provocado por sus interacciones con el contexto (familiar y escolar). La intervención suele enmarcarse en los diferentes contextos en los que vive el alumno/a, o sea, en el ámbito socio-familiar junto con el ámbito educativo.

c) Enfoque "combinado", "interaccionista" $o$ ecléctico. Sostiene que es consecuencia de la interacción entre el contexto y las condiciones propias del alumno/a. La intervención integraría las dos propuestas anteriores (uso de medicación junto a técnicas psicológicas, preferentemente de enfoque cognitivo-conductual).

De todos los enfoques existen estudios con resultados que los avalan y los contradicen, por lo que siendo un tanto escépticos se podría decir que no se conocen realmente su eficacia y eficiencia.

Consideramos que el alumnado con TDAH debe recibir atención tanto dentro como fuera del aula, lo que conllevaría un enfoque multidisciplinar con intervenciones desde la perspectiva médica (con la prescripción farmacológica, si así lo precisase), desde la perspectiva psicológica (con utilización de técnicas de manejo de conducta, de autocontrol, de habilidades de comunicación y de interacción,..), y desde la perspectiva psicopedagógica (para minimizar los retrasos en sus aprendizajes y potenciar la adquisición de competencias académicas en el ritmo de lo esperable para cualquier otro alumno/a de su edad y nivel). Pero aquí surge el gran dilema: ¿cómo se coordina esto?, ¿quién es el responsable de esa coordinación?, ¿con qué recursos se puede contar?...

\section{¿Qué consideramos necesario para la interven- ción en el ámbito educativo?}

Creemos que aquí está parte de la solución al problema. Si consideramos que lo lógico sería un modelo combinado, esto requiere una coordinación entre servicios externos (unidades de salud mental de Sanidad, servicios sociales de Bienestar Social, incluso IMSERSO, -téngase en cuenta que cada comunidad autónoma estos servicios tienen una denominación y una configuración que se pueden organizar y denominar de forma diferente-) y servicios internos (propios del sistema educativo -que a su vez en cada comunidad autónoma funcionan y están configurados de forma diferente-). Igualmente cabría considerar cuál sería la coordinación con los gabinetes o centros privados de tratamiento.

Circunscribiéndonos en un primer momento al mundo educativo, aquí existe una gran dificultad para abordar la problemática de este alumnado -al igual que pasa con otros muchos problemas de esta índole- ya que la formación de los profesionales educativos en este caso concreto suele ser bastante deficitaria, pero es que además no se dispone de recursos técnicos ni materiales -existiendo bajos presupuestos para orientación, escasez de horarios para atención específica, multitud de objetivos a abordar, dispersión de tareas,...--

Si nos circunscribimos al ámbito de los profesores, podemos afirmar de forma rotunda que en sus planes de formación inicial no reciben una formación básica para abordar las necesidades del alumnado con una problemática especial.

Si nos circunscribimos al ámbito de la orientación, las perspectivas no son mucho mejores, ya que la formación inicial tampoco les da herramientas para este abordaje, pero además se ve agravado desde dos puntos de vista: uno el legal, ya que los servicios de orientación no pueden hacer abordaje terapéutico, quedando reglamentado legalmente para el ámbito sanitario -unidades de salud mental-. $\mathrm{Y}$ otro formativo, ya que el perfil formativo del orientador puede de psicólogo, pedagogo o psicopedagogo, condicionando su formación inicial la perspectiva de abordaje de estos casos.

Por otro lado, es necesario hacer un adecuado análisis funcional de conducta, ya que mediante este procedimiento se podrán identificar las situaciones en las que se muestra una conducta concreta 
(variables estimulares antecedentes), las características objetivas de cada comportamiento en cuestión, o sea, las conductas-problema (de una forma operativa y funcional, y teniendo en cuenta su intensidad y frecuencia), y valorando sus componentes motor, emocional y cognitivo, que conllevan unas consecuencias positivas o negativas para el que lo hace, $\mathrm{y}$ unas contingencias de reforzamiento (siempre, casi siempre, a menudo, nunca,...). Y todo esto, teniendo en cuenta la historia de aprendizaje previa, los factores disposicionales del medio y los factores disposicionales del alumno/a.

¿El por qué de todo esto?, ¿cuál es su utilidad? Podemos decir que, sobre todo, para explicar fundamentalmente a los "adultos" cómo se producen determinados comportamientos y cómo se mantienen. Si se consigue que lo "entiendan" esto facilitará, sin duda, la intervención para el cambio y la educación, que es lo que se pretende con dicha intervención.

\section{Propuestas metodológicas para la intervención en} el ámbito educativo con profesores y con padres

Llegados aquí, cabe preguntarse: ¿con qué enfoque se puede o debe intervenir en el ámbito educativo?, ¿teniendo en cuenta el contexto, qué podemos aplicar?, ¿qué requisitos requiere este tipo de intervención?, ¿quiénes estarán implicados?...

Pues bien, desde el ámbito psicoeducativo, podemos hablar de diferentes enfoques o corrientes:

a) Enfoque Conductual, cuyo objetivo principal de tratamiento es la formación de padres y profesores en conocimientos generales y procedimientos, que en su gran mayoría derivan de los principios del condicionamiento operante (técnicas de modificación de conducta), con el fin de comprender y dirigir mejor los problemas de comportamiento en casa y en el colegio (Weiss y Hetchtman, 1992). De esta forma, el comportamiento del alumno/a se va conformando en función de las consecuencias que le siguen. Este aspecto es bastante impor- tante ya que el trastorno a menudo se asocia con alteraciones en la interacción entre padres e hijos.

b) Enfoque Cognitiva, cuyo objetivo principal de tratamiento es enseñarles técnicas de autocontrol y resolución de problemas, debido a que el déficit de autocontrol se considera como un aspecto central en este tipo de trastornos. Las técnicas o procedimientos más utilizados son: técnicas para aumentar la autorregulación, administración de autorrecompensas y autoinstrucciones (Meichembaum y Goodman, 1971).

c) Enfoque Cognitivo-conductual, que comparte y combina los objetivos y procedimientos de los dos enfoques anteriores.

Dentro del enfoque conductual, y respecto a las técnicas de modificación de conducta, a modo de síntesis podemos decir lo siguiente:

La modificación de conducta constituye un conjunto de técnicas de intervención educativa, que se fundamentan en los principios de la teoría del aprendizaje, y cuya finalidad principal es "cambiar la frecuencia o intensidad con que una persona exhibe una determinada conducta". Se ha constatado que la modificación de conducta es especialmente aplicable a los alumnos de educación infantil y primer ciclo de primaria, si bien es aplicable a lo largo de toda la enseñanza primeria, incluso en los inicios de la secundaria, donde hemos constatado que los acuerdos de conducta son eficaces. Además, a estos alumnos "pequeños" aún no se les puede aplicar técnicas de autorregulación de forma sistemática, ya que no suelen tener el desarrollo cognitivo necesario; otra consideración es que el alumnado necesita de una ayuda o guía externa, preferentemente del profesor o terapeuta, para que le guíe en la valoración de cuáles son las conductas adecuadas en la escuela. Entre las técnicas que cabe utilizar están el manejo de contingencias, el reforzamiento diferencial, el moldeamiento, la atenuación de ayudas, el modelado, la práctica negativa, el aislamiento (tiempo sin reforzadores), la restricción de estímulos, la saciedad de estímulo, el coste de res- 
puesta, el contrato de conducta o acuerdo de conducta con economía de fichas y/o coste de respuestas.

También es recomendable el uso del refuerzo verbal como técnica para incrementar o potenciar una determinada respuesta en el alumnola (positivo - estímulo o suceso ambiental cuya aparición, como consecuencia de cierta respuesta, aumenta la probabilidad de aparición o de mantenimiento de ésta en el futuro- y negativo -estímulo o suceso ambiental cuya retirada, como consecuencia de cierta respuesta, aumenta la probabilidad de aparición o mantenimiento de ésta en el futuro), para reforzar e incrementar la conducta realizada (la alabanza o felicitación, debe conllevar el refuerzo verbal con frases como "fenomenal", "lo estás haciendo estupendamente", "sigue así", "muy bien, "buen trabajo", "estupendo", "lo has hecho fenomenal", y junto a la descripción de la conducta positiva; así mismo es eficaz el manejo de contingencias y los contratos de economía de fichas y/o coste de respuestas. De la misma manera las técnicas de modificación de conducta sirven para reducir o eliminar conductas no deseadas, para ello se dispone de técnicas como el aislamiento y el costo de respuesta. Otra consideración a tener en cuenta es que el refuerzo pierde eficacia cuando no se describe la conducta positiva, o sea, lo que ha hecho bien, ya que a veces el alumno/a no suele ser consciente de ello, o cuando se hace referencia a una conducta negativa que sucedió con anterioridad, por ejemplo, si se dice al alumno/a "muy bien, es la primera vez que terminas los deberes en clase", "es la primera vez que traes los deberes de casa", "ya es hora que traigas todos los materiales", "por una vez lo tienes todo,...". En cuanto a la extinción se pretende reducir la frecuencia o intensidad de una conducta; pero hay que tener en cuenta que la retirada absoluta de reforzadores que mantengan una respuesta determinada hará perder a ésta su valor funcional, consiguiendo la extinción progresiva de dicha conducta. Así pues, la extinción, consiste en retirar cualquier tipo de atención ante una conducta no deseada por parte del alumno/a; siendo esta técnica útil para reducir conductas inade- cuadas que los alumnos realizan esperando una respuesta por parte del profesor.

Como ya hemos indicado, el refuerzo consiste en conceder al alumno/a una recompensa cuando realiza una conducta adecuada. Normalmente, esta recompensa suele ser un elemento material reforzante para el alumno/a o un privilegio (por ejemplo borrar la pizarra, recoger cinco minutos antes, controlar la organización de algo en clase, etc.). Para ser eficaces, estos privilegios deben ser motivadores para los alumnos e incluso suponer un reto o un estímulo para presentar un buen comportamiento; deben presentarse inmediatamente después de la realización de la conducta positiva (pierden eficacia progresivamente cuando se alejan temporalmente de la realización de la conducta); y deben ser variados, para evitar que pierdan atractivo para los alumnos. La técnica contraria al refuerzo sería el coste de respuesta (o pérdida de privilegios), que consiste en retirar al alumno/a un refuerzo (por ejemplo, reducir unos minutos el patio) con el objetivo de reducir una conducta no deseada. El costo de respuesta se rige por los mismos principios de eficacia que el refuerzo.

Por último, cabe hacer algunas consideraciones sobre el uso del castigo, que es un proceso cuya finalidad es eliminar la frecuencia de aparición de una respuesta determinada, consistente en la administración de un estímulo aversivo o en la retirada de un estímulo apetitivo cada vez que se ejecuta la misma. Existe dos tipos de castigo, el denominado castigo positivo, que consiste en la presentación de un estímulo aversivo (por ejemplo, un castigo físico, una palabra desagradable, una copia de frases, una mirada terrible, etc); y el castigo negativo, que consiste en la pérdida o retirada de un privilegio, ya sea de un objeto agradable, o de una actividad apetitosa, o de una situación concreta (por ejemplo, dejar sin recreo, no dejar participar en una actividad, perder algo que tiene, etc...). De todos es conocido los inconvenientes del castigo, sin embargo, en algunos contextos puede ser útil y necesario. Téngase en cuenta que el uso general que se hace del castigo no suele ser coincidente con cómo se usaría como téc- 
nica, o dicho de otra forma, se suele utilizar de forma inadecuada para el fin que se debería perseguir.

\section{b y c) Respecto a las técnicas cognitivo-conduc- tuales.}

A modo de síntesis podemos decir lo siguiente:

Las técnicas cognitivo-conductuales son una combinación de los principios anteriores con estrategias de enfoque cognitivo. Desde este enfoque no sólo se intenta eliminar o reforzar conductas, sino que además se trata de influir en el procesamiento cognitivo del alumno/a para que en el futuro sea él quien autorregule su comportamiento, sin necesidad de la presencia de un adulto que aplique la administración de refuerzos o retirada de los mismos. Estas técnicas son especialmente útiles para alumnos a partir del segundo ciclo de educación primaria, ya que van adquiriendo un desarrollo madurativo suficiente como para autorregular su comportamiento. Las estrategias cognitivo-conductuales más habituales son las autoinstrucciones y la autoevaluación reforzada.

El procedimiento de las autoinstrucciones de Meichenbaum, consistentes en enseñar al alumno/a a decirse a sí mismo qué es lo que tiene que hacer para afrontar una tarea determinada, siguiendo una secuencia de instrucciones. Estas secuencias autoinstruccionales suelen constar de tres fases: planificación, automonitoreo, y autocomprobación. En la práctica, el procedimiento a seguir básicamente sería un entrenamiento auto-instruccional empleando tarjetas de auto-instrucciones. Las fases serían:

1. El profesor/terapeuta propone al alumno/a una lista de frases referidas a su conducta problema. Dichas frases pretenden constituir un elemento de auto-control cognitivo.

2. Una vez que el alumno/a las acepta como razonables y aplicables a su situación, las memorizará.

3. Cuando el profesor/terapeuta ha comprobado que las conoce de memoria se procederá a efectuar una simulación de las diversas situaciones y el empleo de las frases citadas. Con alumnos pequeños, el asesor utilizará técnicas de modelado, según va verbalizando en voz alta las autoinstrucciones necesarias. En un segundo momento, se le pedirá al alumno/a que las verbalice en voz alta, después en voz baja, y, por último, en silencio. Con alumnos de más edad, no es tan necesario que el profesor/terapeuta actúe como modelo, pidiendo directamente al alumno/a que lo haga como en el caso anterior.

4. Posteriormente se supervisa el empleo de las frases en las situaciones reales y los resultados obtenidos por el interesado. Es importantísimo que el profesor/terapeuta llame la atención del alumno/a tanto respecto a las consecuencias naturales de su nueva conducta, como de su estado emocional cuando actúa de esta manera. Sólo cuando el alumno/a se percata de los reforzadores naturales de este nuevo modo de pensar cabe esperar un mantenimiento y una generalización del procedimiento.

Estas técnicas no son exclusivas para TDAH, valdrían para potenciar los aprendizajes de cualquier otro alumno/a. Existen diversos programas que adaptan la secuencia autoinstruccional primitiva de Meichenbaum y Goodman (1971) a alumnos de diferentes edades; en estos programas suelen presentarse las autoinstrucciones en forma de apoyos gráficos que recuerdan al alumno/a cada una de las verbalizaciones que debe decirse a sí mismo. Estos apoyos gráficos se emplean hasta que el alumno/a finalmente interioriza las autoinstrucciones, y las incluye en su propio repertorio de estrategias cognitivas (Miechenbaum, 1981; Miechenbaum y Goodman, 1969, 1971).

Consideramos que la intervención en TDAH se debe realizar desde una doble perspectiva, desde la intervención psicológica y desde la intervención psicopedagógica.

Desde la perspectiva de la intervención psicológica, habría que considerar los siguientes elementos:

- Asesorar, y "formar-guiar" apoyando a los padres y profesores durante el proceso de ayuda al hijo/alumno.

- Diseñar planes de acción específicos para cada alumno/a, que incluyan al alumno/a TDAH, a 
sus padres y a ser posible a otros familiares, si así se considera procedente, que convivan con él habitualmente, así como a sus profesores y compañeros.

- Supervisar y/o aplicar el programa específico de entrenamiento.

- Mantener contacto y colaboración con el resto de profesionales implicados.

Desde la perspectiva de la intervención psicopedagógica, habría que tener muy en cuenta otra serie de consideraciones previas antes de hablar de técnicas y de formación. Nos referimos al estilo de enseñanza-aprendizaje.

Previamente a las diferentes técnicas que se suelen emplear en el abordaje de estos casos, creemos que procede realizar una reflexión sobre el estilo de enseñanza-aprendizaje, lo cual equivale a hablar de las condiciones del aula, que en su mayor parte son impuestas por el profesor, y las maneras en que los alumnos se desenvuelven en la misma. Los alumnos poco a poco aprenden a preferir aquellas condiciones que tienen relación con sus éxitos, y a rechazar aquellas otras ante las cuales fracasan, lo cual, unido a sus creencias sobre su manera de ser, es decir, aquello que hace que exista una cierta interpretación de las circunstancias dadas, genera un modo de comportarse habitualmente.

Desde nuestra perspectiva, el estilo de aprendizaje no debe ser confundido con las habilidades o destrezas que el alumno/a posee o dicen poseer; nos referimos exclusivamente a cómo un alumno/a responde cuando tiene ante sí la posibilidad de hacer las cosas de varias maneras diferentes, y a cómo responde ante los diferentes ambientes de aprendizaje a que se ve sometido en la práctica escolar diaria, sin posibilidad de elegir. En él caben tres dimensiones: la cognitiva, la físico-ambiental y la socioafectiva y motivacional.

La evaluación del estilo de aprendizaje de un alumno/a en estos niveles se efectúa principalmente a través del juicio del profesor, y como cada profesor manifiesta a su vez un estilo propio de enseñanza, diseñando en función de él un ambiente instruccional determinado, difícilmente sabrá qué haría su alumno/a en otras circunstancias; pero si ante los diferentes tipos de interacciones educativas que se dan en el aula, en función del estilo de enseñanza establecido, la respuesta del alumno/a no es la adecuada, siempre le quedará al profesor el recurso de cambiar tales circunstancias y probar si su comportamiento mejora.

Aquí radica la importancia de la evaluación de estilos, educativo y de aprendizaje, en la posibilidad de ponerlos frente a frente y constatar hasta qué punto se están ofreciendo a los alumnos, y especialmente a aquellos que presentan dificultades de aprendizaje, los ambientes y circunstancias más adecuadas. Al hacerlo debemos prestar atención a los siguientes aspectos:

- ¿Cómo se enfrenta el alumno/a a los procesos generales de enseñanza-aprendizaje?

- ¿Cómo se enfrenta a diversas tareas específicas?

- ¿Cómo se enfrenta a los diferentes tipos de materiales?

- ¿Cómo responde ante los diferentes sistemas de agrupamiento?

- ¿Cómo responde ante las ayudas que se le prestan?

Para nosotros está claro que la escuela, tal y como la conocemos, potencia unos determinados estilos en detrimento de otros. Por ello, no debe resultarnos extraño que aparezcan en la clase estilos de aprendizaje parecidos, pero, también otros diferentes a los que hay que atender, potenciar, y si es preciso, modificar.

Unos sencillos instrumentos y una alta dosis de honestidad son suficientes para la evaluación del estilo propio y el de los alumnos. Por eso cabe preguntarse: ¿por qué esperar a que nos digan cómo deberían cambiar nuestras clases y las dinámicas con las que se desarrollan?

Como ya se ha dicho, consideramos que el objetivo de un programa de intervención requiere los siguientes componentes:

a) Formación de docentes.

b) Formación de padres.

c) Intervención directa con el alumnado. 
Desde el punto de vista de la intervención, consideramos que hay que abordar los siguientes ámbitos:

a) Ámbito comportamental.

b) Ámbito de los aprendizajes.

c) Ámbito emocional.

d) Ámbito social.

a) Desde el punto de vista comportamental:

1. A partir del entrenamiento para la mejora de las relaciones sociales, se deberán adquirir habilidades cognitivas de solución de problemas interpersonales ("enseñar a pensar"), así como en habilidades de comunicación y en habilidades para regular y controlar las emociones (asertividad, autoestima, ansiedad, estrés, ira, frustración, miedo,...) Controlar las "llamadas de atención", dispensando una atención selectiva a los alumnos con déficit en su comportamiento; para ello, se debe dispensar refuerzos ante conductas positivas, mostrarles agrado, llamarlos a menudo a la mesa del profesor para mostrarles una atención y refuerzo verbal positivo, recorrer la clase prestando la atención y refuerzo selectivo y adecuado; observar cómo trabajan y valorar sus evoluciones, ...

2. Facilitar el movimiento por el espacio del aula, pero controlando que no resulte disruptivo para los demás compañeros, y sobre todo, reforzar los comportamientos correctos. Facilitar la segmentación de tareas por partes, de forma que pueda obtener éxitos parciales que le lleven al global.

3. Las recriminaciones se le deben hacer en privado. Aplicando sólo excepcionalmente medidas disciplinarias (véase tabla 1 y $2^{*}$ ).

4. Entrenamiento en aspectos de control emocional, irritabilidad, agresividad, autoconcepto, autoestima, asertividad...

b) Desde el punto de vista de los aprendizajes:

Numerosas investigaciones han constatado en las últimas décadas los efectos beneficiosos de las inter- venciones psicopedagógicas basadas en técnicas conductuales y cognitivo-conductuales en la escuela (una revisión exhaustiva de estos trabajos puede encontrarse en el trabajo elaborado por Miranda, Jarque y Tárraga, 2006). A continuación se exponen brevemente las potencialidades y aspectos más positivos de este tipo de intervenciones:

Tanto las intervenciones conductuales como las cognitivo-conductales se han mostrado consistentemente eficaces en la mejora de la adaptación escolar en los estudiantes con TDAH. Las técnicas de modificación de conducta presentan buenos resultados a corto plazo, ya que la aplicación sistemática adecuada de contingencias tras la conducta de los alumnos produce la desaparición de conductas inadecuadas y el reforzamiento de conductas deseadas. Igualmente, las estrategias cognitivo-conductuales son positivas, ya que ayudan a los alumnos a controlar por sí mismos su propia conducta, autorregulando su comportamiento y autoevaluando si éste es o no adecuado.

Ambos tipos de intervenciones tienen la ventaja de que apenas consumen tiempo para su correcta aplicación; tan sólo las estrategias cognitivo-conductuales requieren dedicar algunas sesiones a la enseñanza y práctica de las autoinstrucciones o de la autoevaluación. Sin embargo, una vez que el alumno/a ha aprendido e interiorizado estas estrategias, las aplica sistemáticamente sin apenas consumir tiempo dedicado a los contenidos curriculares, ya que estas estrategias se convierten en una rutina casi automática en la que el alumno/a realiza los procedimientos autónomamente, con supervisión esporádica por parte del profesor. Este modo de funcionar hace que ambos procedimientos sean "económicos" en cuanto a consumo de tiempo, ya que invertir algunas sesiones en enseñar al alumno/a a autorregular su comportamiento requiere mucho menos tiempo que tener que interrumpir las clases continuamente para corregir la conducta del alumno/a.

Establecer una línea base en sus aprendizajes, y a partir de ellas desarrollar la adquisición de competencias instrumentales básicas a través del dominio de aquellos contenidos o conocimientos que aún no 
tienen adquiridos, y que como alumnos con necesidades educativas son susceptibles de programas de trabajo individual o adaptaciones curriculares. Desde el punto de vista de los déficits de atención procedería diseñar intervenciones tendentes a la mejora de la capacidad de atención, mediante el entrenamiento en actividades en focalización $\mathbf{y}$ mantenimiento de la atención. Así, para alumnos pequeños se pueden realizar ejercicios de rompecabezas de letras y figuras geométricas sencillas, de punteo, de reconocimiento de errores en dibujos sencillos, de localización de dibujos repetidos. Mientras que para alumnos más mayores se podrían utilizar sopas de letras, de reproducción de figuras mediante números, de reconocimiento de errores en dibujos complejos, de crucigramas, de completar frases o palabras.

Como pautas para abordar los déficits de atención debe tenerse en cuenta el diseño de actividades cortas, atractivas y diversificadas, llegando a fragmentar en parte las tareas que sean más largas. Asimismo, es útil la utilización de agendas o libretas de anotaciones para facilitarles la organización y que vayan aprendiendo a planificar su actuación dentro y fuera del aula. También hay que dispensarles una atención selectiva, sin que llegue a causar rechazo por parte de sus compañeros, o que incite a sus compañeros a seguir sus mismos pasos (negativos).

Otras actuaciones muy importantes son las de facilitar la ayuda de especialistas dentro del centro (si es que existen tales "especialistas"), así como la de facilitar y promover la coordinación con especialistas externos al centro educativo. ¿Cómo y cuándo...?

Desde el punto de vista de la intervención pedagógica directa sobre el alumno/a con TDAH hay que tener muy en cuenta que por sus característica suelen tener dificultades de comprensión de instrucciones, tanto orales como escritas, lo que conlleva respuestas erróneas, incompletas o incorrectas. Por ello, el profesor debe tener en cuenta una serie de consideraciones, como son: repetirle las instrucciones o enunciados un par de veces; a continuación pedirle que le repita lo que se le ha dicho o pedido. Si la instrucción o tarea es por escrito debe pedirle que le explique lo que se le pide, qué es lo que tiene que hacer; a continuación preguntarle cómo va o debe de hacerlo, explicando por qué lo va a hacer de esa manera, para a continuación supervisar cómo lo va haciendo y analizar con él el resultado.

El entrenamiento en autoinstrucciones verbales conllevaría el uso de técnicas como modelado, guía externa manifiesta, autoguía y autoinstrucciones manifiestas. En el modelado, se le irán diciendo los pasos a seguir, mediante unas instrucciones claras (por parte del modelo, en este caso sería el propio profesor) que el alumno/a deba seguir aplicando, es lo que denominamos guía externa (repitiéndose el alumno/a, mediante autoinstrucciones, las instrucciones que ha visto en el "profesor-modelo"), pasando a un mayor nivel de complejidad haciendo de sus verbalizaciones una autoguía en voz alta, para después pasar a una fase de autoguía manifiesta atenuada ("cuchicheo"), para terminar con las autoinstrucciones encubiertas, momento en que el alumno/a deberá realizar la tarea en silencio mientras se guía por las instrucciones a través del pensamiento. Es lo que sería un proceso en el que se va haciendo atenuación de ayudas.

En cuanto a la ubicación en el aula, habría que controlar el espacio físico, teniéndole sentado, o bien solo, o con un compañero con buen rendimiento y habilidades sociales. Preferentemente cerca del profesor (aunque no pegado a su mesa, para evitar la distracción), en una zona tranquila, lejos de puertas y ventanas -manejo de contingencias-; se debe controlar la distancia entre las mesas ( $;$ si el espacio lo permite!), buscando en silencio "ambiental" cuando el tipo de trabajo lo requiera y permita. Y sobre todo, eliminar los posibles estímulos distractores (ya que algunas clases son un collage de elementos distractivos colgados en sus paredes, que posibilitan la distracción de cualquier alumno/a, y de forma más específica a los TDAH y a los alumnos con bajo rendimiento y/o fracaso escolar).

Otras consideraciones a tener en cuenta son que hay que ser muy metódico y constante en la utiliza- 
ción de los sistemas de reforzamiento, tratando de reforzar el trabajo acabado y sobre todo el trabajo bien acabado; hacer un constante feedback, para informarle de sus mejoras; al menos en las situaciones iniciales hay que tratar de evitar los fracasos. Y a la hora de diseñar el programa de intervención se deben priorizar los aspectos alterados: atención, razonamiento, comprensión, habilidades sociales, memoria... Así pues, para que un programa educativo tenga éxito se debe adecuar las exigencias escolares a las capacidades y características del alumno/a, partiendo de sus puntos fuertes para que empiece obteniendo éxitos. Así mismo hay que mostrarle unas reglas de funcionamiento claras y realistas, haciéndolas visibles para que las vean con facilidad y frecuencia; generar un ambiente de trabajo y orden dentro del aula, con uso frecuente de agenda para anotar las actividades y tareas que se van a realizar.

\section{c) Desde el punto de vista emocional:}

1. Potenciar la asertividad, autoconcepto y austoestima, favoreciendo la elaboración objetiva de sus errores, sin hacerle sentirse culpable o rechazado. Es importante posibilitar una retroalimentación positiva. Eliminar o reducir los niveles de ansiedad y estrés.

2. Evitar valorar siempre negativamente al alumno/a.

3. Generar expectativas de éxito futuro.

4. Permitir la retroalimentación de forma que sea consciente de sus avances.

\section{d) Desde el punto de vista social:}

1. Ayudar al alumno/a a controlar su conducta situando su ubicación en el aula próxima al profesor.

2. Evitar una percepción, por parte de sus compañeros, como una conducta agresiva hacia ellos o como una conducta impulsiva intencionada.

3. Evitar el etiquetaje moral ("eres malo", "eres...", "eres...") y ayudarles a interpretar adecuadamente cada situación conflictiva.
4. Favorecer que sus compañeros aprendan a darle oportunidades para integrarse en el juego y en otras dinámicas de la clase-aula, y tratando de ignorar sus comportamientos inadecuados.

5. Asesorar a los padres con información que les sea útil para fomentar sus interacciones positivas y su integración.

En conclusión, las técnicas de modificación de conducta y las estrategias cognitivo-conductuales son una herramienta eficaz para la mejora de la adaptación escolar de los estudiantes con TDAH -al igual que para cualquier otro alumno/a-, por lo que su aplicación en el aula es muy recomendable. Estas estrategias pueden iniciarse en el "aula de pedagogía terapéutica" -dependiendo de la singularidad de la organización de los apoyos en cada centro-, y posteriormente pueden generalizarse al resto de contextos escolares, con una adecuada coordinación de todos los implicados, ya que es importante que los alumnos perciban que las normas son claras y coherentes en todas las aulas.

Pero cabe preguntarse: ¿es esto suficiente para dar respuesta a la problemática de estos alumnos?, ¿tiene la "escuela actual" las condiciones para llevar estas teorías a cabo?, ¿es factible el abordaje en el contexto actual de la escuela?....

Otras consideraciones a tener en cuenta en el aula serían las siguientes:

En los casos de hiperactividad se debe tener cierta flexibilidad cuando se vea que se levanta, y haya ha terminado la actividad, mandarle a realizar "recados" o cualquier actividad que precise movimiento para que pueda "soltar" energías. Asimismo, cuando se vea que está inquieto sugerirle salir de clase y darse una vuelta, esto bajará la tensión y volverá más tranquilo, pero ¿qué ocurriría si al salir ocurriese un accidente"?. Esto está bien desde el punto de vista terapéutico, pero choca con la parte legal.

En los casos de impulsividad, es aconsejable la utilización del modelo de autoinstrucciones y del sistema de autorregulación, desarrollando el entrenamiento en habilidades sociales (pero, ¿cuándo, en qué espacio físico y temporal?), y sobre todo, no 
enfadarse con ellos sino proponerles alternativas (estamos educando y formando).

Por último, por lo general en nuestro sistema educacional nos fijamos sobre todo en lo negativo. Mirando fríamente, casi siempre, se observan en estos alumnos algún aspecto positivo como espontaneidad, creatividad, sinceridad, innovación, energía, buen humor, intuición, lealtad, etc.; pero ¿se utilizan para favorecer sus aprendizajes?

A continuación esbozaremos de forma sintética los componentes que consideramos necesarios en las propuestas de formación de profesores y padres.

\section{- Propuesta de plan de actuación con docen-} tes:

1. Definición de objetivos, contenidos y procedimientos de la formación y de la intervención.

2. Conceptualización sobre aspectos diagnósticos, etiología, datos epidemiológicos, evolución del trastorno, problemas y sintomatología asociada, estrategias de tratamiento, necesidades educativas y algunas experiencias previas.

2. Entrenamiento en técnicas de modificación de conducta: utilización de reforzadores (sociales, materiales, verbales y no verbales), conocimiento del principio de Premack, la ley del efecto, la ley del ejercicio, la ley de la disposición, sistema de economía de fichas y/o coste de respuestas, tiempo fuera (aislamiento), extinción, y otras técnicas de modificación de conducta.

4. Entrenamiento en técnicas cognitivo-conductuales: como autoinstrucciones, técnicas de autocontrol y técnicas de autoevaluación reforzada (Miechenbaum, 1981; Miechenbaum y Goodman, 1969, 1971).

5. Entrenamiento en técnicas de resolución de problemas: programas de habilidades de interacción social (identificar el problema, definirlo, explorar alternativas, actuar con un plan y evaluar los logros alcanzados) (Fernández, 2000; Vallés, 2008).
6. Entrenamiento en habilidades sociales: consistentes en habilidades para hacer preguntas, para escuchar, colaborar, buscar ayuda, autorreforzarse, pedir disculpas, dar las gracias, conversaciones, hacer y recibir críticas y cumplidos, ofrecer ayuda y expresión de sentimientos. (Goldstein, Sprafkin y Klein, 1976; Goldstein, Sprafkin, Gershaw y Klein, 1989; Michelson, Sugai, Wood y Kazdin, 1987; Monjas, 1996).

7. Entrenamiento en técnicas de reflexividadimpulsividad: para demora forzada, estrategias cognitivas de escudriñamiento y análisis de detalles, autoinstrucciones, modelado y reforzadores (Gargallo, 1996, 2000).

8. Estudio de casos (a través de dinámicas de discusión de grupos, rol-playing, visualización de algunos materiales en video, autoaplicación de algunos materiales, etc. (Galve, 2008, Planas, 2008).

9. Diseño de planes de trabajo individual o de adaptaciones curriculares (Galve y Trallero, 2003).

10. Evaluación, seguimiento y feedback de la formación. Seguimiento de la intervención.

\section{- Propuesta de plan de actuación con padres}

Algo similar se suele hacer con los padres, ya que la escuela puede realizar funciones de formación a padres de modo sistemático, mediante las escuelas de padres, o de modo más puntual, mediante las entrevistas con los especialistas en pedagogía terapéutica o con los psicopedagogos.

Esta formación debe basarse en explicar a los padres mediante ejemplos prácticos diferentes técnicas de manejo del comportamiento en casa, aplicando las estrategias conductuales y cognitivo-conductuales expuestas anteriormente a situaciones familiares; enseñando procedimientos para ayudar a sus hijos a completar las tareas escolares; o simplemente ofreciendo información sobre los síntomas y el pronóstico del trastorno. 
Esta formación es crucial, dado que en muchas ocasiones los padres no tienen un conocimiento profundo del TDAH, y en ocasiones esta formación no es totalmente rigurosa, y proviene de fuentes no científicas que pueden inducir a errores.

Igualmente, muchos padres desconocen que el TDAH puede abordarse no sólo desde una perspectiva psicopedagógica, sino también desde un punto de vista clínico-médico. Por ello, desde la escuela debe informarse a los padres que es conveniente consultar con los servicios médicos especializados (médico de familia y posteriormente servicio de neuropediatría), ya que en ocasiones estos servicios proponen una intervención farmacológica que en combinación con la intervención psicopedagógica puede ofrecer resultados muy positivos.

De forma resumida, el programa a desarrollar básicamente sería el siguiente:

1. Definición de objetivos, contenidos y procedimientos de la formación y de la intervención.

2. Conceptualización sobre aspectos diagnósticos, etiología, datos epidemiológicos, evolución del trastorno, problemas y sintomatología asociada, estrategias de tratamiento, necesidades educativas y algunas experiencias previas.

3. Entrenamiento en técnicas conductuales y cognitivo-conductuales.

4. Análisis funcional de las relaciones con los hijos: entrenamiento en habilidades sociales y resolución de problemas (ayuda con deberes escolares). Implicación de los padres en el aprendizaje de los hijos.

5. Evaluación y seguimiento de la formación. Seguimiento de la intervención.

En cuanto a la metodología que con frecuencia se ha usado, preferentemente ha sido mediante estudio de casos, rol-playing, dinámicas de grupos, visualización de videos, autoaplicación de algunos materiales, lluvia de ideas, debates, etc. Todo ello se puede dinamizar mediante técnicas de dinámica de grupos. Cobra mucha importancia la preparación de reuniones con padres; ya que una entrevista bien dirigida puede conllevar una evolución adecuada del proceso a seguir; y viceversa.
También es interesante realizar una tutoría guia$d a$ de padres, bien desde los servicios externos o internos al centro educativo.

Pero, de nuevo nos surgen las mismas preguntas: ¿quién debe o puede o tiene que hacer esta formación?, ¿es esto suficiente para dar respuesta a la problemática de estos alumnos?, ¿tiene la "escuela actual" las condiciones para llevar estas teorías a cabo?, ¿es factible el abordaje en el contexto actual de la escuela?....

\section{Discusión, propuestas y conclusiones}

Desde estos enfoques existen estudios publicados, en los que se trata de evidenciar la eficacia del tratamiento, pero desde nuestra perspectiva cabe una crítica, y es que son estudios que podríamos denominar de "laboratorio", donde las circunstancias y el contexto están controlados, dándose los medios para su desarrollo; pero aquí viene la gran pregunta: ¿se puede generalizar esta forma de actuar la contexto educativo general, o actual?; ¿en un centro, que exista, uno, dos, tres casos, se puede poner en marcha este tipo de estrategias de intervención?, en caso afirmativo, ¿con qué recursos, con qué profesionales se puede contar, con qué costos, en qué tiempos,...?

A modo de ejemplo, se pueden nombrar los trabajos con tratamientos cognitivo-conductuales (Abikoff, 1991; Baer y Nietzel, 1991; Calderón, 2001; Miranda, Presentación, Gargallo, Soriano, Gil y Jarque, 1999; Miranda, Soriano, Presentación y Gargallo, 2000), donde se han puesto de manifiesto la eficacia de este tipo de intervenciones, aunque no siempre los resultados han sido satisfactorios. Sólo se suele observar una evolución positiva cuando se abordan de forma simultánea los aspectos neurofisiológicos/clínicos, familiares, sociales y escolares.

Pero volvemos a decir, ¿son extrapolables al contexto educativo actual en nuestro país? Francamente, nuestra experiencia nos dice que no.

En diferentes estudios se constata que la intervención farmacológica tiene sus ventajas y limitaciones para abordar a plenitud un cuadro aparentemente cró- 
nico como el TDAH. El tratamiento psicoestimulante aparece óptimo para tratar los síntomas centrales del TDAH, sin embargo, parece deficiente a la hora de tratar sus síntomas asociados, y en muchos casos tiene otros efectos secundarios o colaterales. Por otro lado, la intervención multicomponente sumada a la medicación parece proporcionar un manejo más adecuado para tratar los síntomas asociados al TDAH y mejorar el funcionamiento global en los alumnos en edad escolar, pero es incapaz de manejar eficazmente los síntomas centrales del desorden.

Finalmente los programas multicomponentes suelen conllevar mejoras significativas en el funcionamiento individual (autoestima, autoconfianza), así como en la relación con padres, en competencia social (habilidades sociales, relaciones interpersonales) y en el funcionamiento escolar (habilidades y hábitos de estudio, actitud hacia los profesores), pero, ¿esto se puede hacer en cualquier centro educativo?
Creemos que no, con la actual formación del profesorado, junto a la organización y prioridades de los centros educativos, lo que no se puede pedir a los profesores de apoyo (por ejemplo, pedagogía terapéutica) es que sean la solución a todos los problemas de los alumnos con dificultades o necesidades, pues por su formación, y por la problemática en cada centro, su intervención está mediatizada; ni tampoco a los orientadores ya que no pueden hacer "terapias" a tenor de la normativa vigente [en muchos casos, sería susceptible la ayuda de profesionales externos, llegando a una actuación combinada si en realidad existiese la coordinación o cumplimentación entre ayudas externas e internas].

Por último adjuntamos unos cuadros síntesis sobre el grado de eficacia de las técnicas (tabla 1) que hemos hablado con anterioridad, junto a otro que valora la influencia del comportamiento de los educadores en la conducta de los alumnos/as (tabla 2).

Tabla 1. Grado de eficaciarelativa de cada uno de los recursos disponibles para el tratamiento de los problemas de relaciones sociales

\begin{tabular}{|c|c|c|c|c|c|c|}
\hline & \multicolumn{6}{|c|}{ Edad de los alumnos } \\
\hline & $1-3$ & $4-5$ & $6-8$ & $9-11$ & $12-14$ & $15-18$ \\
\hline Manejo de contingencias & $* * * *$ & $* * * *$ & $* * *$ & $* *$ & $* *$ & $*$ \\
\hline Resistencia a la frustración & - & $*$ & $* *$ & $* * *$ & $* * *$ & $* * * *$ \\
\hline Demora de la gratificación & - & $* *$ & $* * *$ & $* * *$ & $* *$ & $*$ \\
\hline Reestructuración cognitiva & - & $*$ & $* *$ & $* * *$ & $* * * *$ & $* * * *$ \\
\hline Auto-instrucciones & - & - & $*$ & $* *$ & $* * *$ & $* * * *$ \\
\hline Habilidades previas solución problemas & $*$ & $* *$ & $* * *$ & - & - & - \\
\hline Solución cognitiva problemas interpersonales - & $*$ & $* *$ & $* * *$ & $* * * *$ & $* * * *$ & \\
\hline Relajación & - & $*$ & $* *$ & $* * *$ & $* * *$ & $* * *$ \\
\hline Comunicación asertiva & - & - & $*$ & $* *$ & $* * *$ & $* * * *$ \\
\hline Reorganización ambiental & $* * * *$ & $* * * *$ & $* * *$ & $* * *$ & $* *$ & * \\
\hline
\end{tabular}

Tabla 2. Influencia del comportamiento de los educadores en la conducta de los alumnos/as $(*)$

\begin{tabular}{|c|c|c|c|}
\hline & \multicolumn{3}{|c|}{ Procedimiento } \\
\hline & $\begin{array}{c}\text { Efecto \% } \\
\text { Mejora }\end{array}$ & $\begin{array}{l}\text { Efecto \% } \\
\text { Indiferente }\end{array}$ & $\begin{array}{l}\text { Efecto \% } \\
\text { empeora }\end{array}$ \\
\hline Recriminación Pública & 40 & 13 & 47 \\
\hline Recriminación Privada & 83 & 10 & 7 \\
\hline Conversación particular elogiosa y estimulante & 96 & 4 & 0 \\
\hline Elogio Público & 91 & 8 & 1 \\
\hline Sarcasmo en Público & 10 & 13 & 77 \\
\hline Sarcasmo en Particular & 18 & 17 & 65 \\
\hline Reconocimiento Público de que el alumno/ ${ }^{a}$ está mejorando & 95 & 4 & 1 \\
\hline Manifestación Pública de que el alumno/ ${ }^{a}$ está empeorando & 6 & 27 & 67 \\
\hline
\end{tabular}

(*) Experiencias efectuadas por BRIGS. Tomado de Espinar Bellón, Andrés: Manual Técnico del tutor. Ed. Ágora. Málaga. 1989, pág 104 


\section{Referencias}

Abikoff, H. (1991). Cognitive training en ADHD children: less to it than meets the eye. Journal of Learning Disabilities, 24, 205-209.

Armstrong, T. (2001). Síndrome de Déficit de Atención con o sin Hiperactividad. Estrategias en el aula. Paidós.

Baer, R. y Nietzel, M. (1991). Cognitive and behavioral treatment of impulsivity in children: a meta-analytic review of the outcome literature. Journal of Clinical Child Psychology, 20, 400412.

Bauermeister, J.J. (2002). Hiperactivo, Impulsivo, Distraído. ¿Me conoces?. Guía sobre el déficit atencional para padres, maestros y profesionales. Bilbao: ALBOR-COHS.

Beltrán, F.J., y Torres, I. (2003). Programa de Entrenamiento en Habilidades Metacognitivas para maestros de niños hiperactivos. Bilbao: COHS

Brown, T. (2003). Trastornos por déficit de Atención y comorbilidades en niños, adolescentes y adultos. Madrid: Masson.

Calderón, C. (2003). Trastorno por déficit de atención con hiperactividad. Programa de tratamiento cognitivo-conductual. Tesis doctoral. Universidad de Barcelona.

Calderón, C. (2001). Resultado de un programa de tratamiento cognitivo-conductual para niño/as con trastorno por déficit de atención con hiperactividad. Anuario de Psicologia, 32 (4), 79-98.

DSM-IV-R-TR (2002). Manual diagnóstico y estadístico de los trastornos mentales. Barcelona: Masson.

Espinar Bellón, A. (1989). Manual Técnico del tutor. Málaga: Ed. Ágora.

Fernández, E. (2000). El método IDEAL para el desarrollo de problemas. En F. Justicia, J.A. Amezcua y M.C. Pichardo (Coords.), Programas de intervención cognitiva (pp. 179-188). Granada: Grupo Editorial Universitario.

Fernández, F.D. (2002). Atención a la diversidad en la escuela inclusiva: pautas e instrumentos para la evaluación e intervención psicoeducativa de alumnos con trastorno por déficit de atención con hiperactividad. En M. Lorenzo et al. (Coords.), $\mathrm{La}$ organización de los nuevos procesos de institucionalización de la educación. Granada: Grupo Editorial Universitario.

Hinshaw, S.P. (1992). Academic underachievement, attention deficits and aggression: comorbidity and implications for intervention. Journal of Consult Clinical Psychology, 60, 893-903.

Galve Manzano, JL., y Ayala, CL. (2001). Evaluación e informes psicopedagógicos. Colección propuestas curriculares. Madrid: CEPE.

Galve, J.L. y Trallero, M. (2003). Adaptaciones curriculares. Madrid: CEPE.

Galve, J.L.(Coord) (2008). Evaluación e Intervención Psicopedagógica en Contextos Educativos. Vol. I: Estudio de casos de dificultades de lenguaje (oral y lecto-escrito). Vol. II: Problemática asociada con dificultades de aprendizaje. Madrid: EOS.

García Pérez, E.M., y Magaz, A. (2003). Mitos, Errores y Realidades sobre la Hiperactividad. Guía para padres y profesionales. Bilbao: COHS.

García Pérez, E.M., y Magaz, A. (2003). Protocolo de evaluación de la hiperactividad. Baracaldo: ALBOR-COHS

García Pérez, E.M., y Magaz, A. (2003). Hiperactividad. Guías para Padres y Maestros. Bilbao: COHS.

Gargallo, B. (1996). La intervención pedagógica en el ámbito de la reflexividad. Un programa educativo para segundo de Primaria. Bordón, 48 (2), 225-238.

Gargallo, B. (1996, 1997, 2000). Programa de intervención educativa para aumentar la atención y la reflexibilidad. PIAAR. Madrid: TEA.

Goldstein, A.P., Sprafkin, R.P. y Klein, P. (1976). Skill training for community living: applying structured learning therapy. New York: Pergamon Press.

Goldstein, A.P., Sprafkin, R.P., Gershaw, N.J. y Klein, P. (1989). Habilidades sociales y autocon- 
trol en la adolescencia. Barcelona: Martínez Roca.

Goldstein, S. y Goldstein, M. (1992). Hyperactivity: why won't my child pay attention. New York: J. Wiley.

Kirby, E.A. y Grimley, A.K., (1992). Fluidez y flexibilidad cognitiva. Bilbao: COHS.

Meichenbaum, D., y Goodman, J. (1971). Training impulsive children to talk to themselves: A measure of developing self control. Journal of abnormal psychology, 77, 115-126.

Miechenbaum, D. y Goodman, J. (1969). Reflection-impulsivity and verbal control of motor behaviour. Child development, 40, 785-797.

Miechenbaum, D. y Goodman, J. (1971). Training impulsive children to talk to themselves: a mean of developing self-control. Journal of Abnormal Psychology, 77, 115-126.

Miechenbaum, D. (1981). Una perspectiva cognitivo-comportamental del proceso de focialización. Análisis y Modificación de Conducta, 7, 85-109.

Michelson, L., Sugai, D.P., Wood, R.P. y Kazdin, A.E. (1987). Las habilidades sociales en la infancia. Barcelona: Martínez Roca.

Miranda, A., Jarque, S., Tárraga, R. (2006). Interventions in school settings for students with ADHD. Exceptionality, 14, 35-52.

Miranda, A. y Santamaría, M. (1986), Hiperactividad y dificultades de aprendizaje. Análisis $y$ técnicas de recuperación. Valencia: Promolibro.

Miranda, Presentación, Gargallo, Soriano, Gil y Jarque (1999). El niño hiperactivo (TDAH). Intervención en el aula. Un programa de formación para profesores. Castellón: Universitat Jaume I.

Miranda, A., Soriano, M., Presentación, M.J., y Gargallo, B. (2000). Intervención psicoeducativa en estudiantes con trastorno por déficit de atención con hiperactividad. Desde la investigación a la práctica. Rev. de Neurología: 38.

Monjas, M.I. (1996, 2004). Programa de enseñanza de habilidades de interacción social para niños y adolescentes (PEHIS). Madrid: CEPE.
Monjas, M.I. (coord.) (2007). Cómo promover la convivencia: Programa de asertividad y habilidades sociales (PAHS). Educación infantil, primaria y secundaria. Madrid: CEPE.

Orjales, I. (1999). Déficit de Atención con Hiperactividad. Manual para padres y educadores. Madrid: CEPE.

Orjales, I (2000). Programa de entrenamiento para descifrar instrucciones escritas. Madrid: CEPE.

Orjales, I. y Polaino-Lorente, A. (2001). Programas de Intervención Cognitivo-conductual para niños con Déficit de Atención con Hiperactividad. Madrid: CEPE.

Planas, J.A.. Evaluación e intervención en alumnado con déficit de atención con hiperactividad : detección y estrategias de intervención en el aula y en la familia, en Galve, J.L.(Coord) (2008). Evaluación e Intervención Psicopedagógica en Contextos Educativos. Vol. I: Estudio de casos de dificultades de lenguaje (oral y lecto-escrito). Vol. II: Problemática asociada con dificultades de aprendizaje. Madrid: EOS.

Polaino, A., Avila, C., Cabanyes, J., García Villamisar, D., Orjales, I. y Moreno, C . (1997). Manual de Hiperactividad Infantil. Unión Editorial.

Rief, S. (1999). Cómo tratar y enseñar al niño con problemas de atención e hiperactividad. Buenos Aires: Paidós.

Trallero Sanz, M. Alumnado con dificultades de aprendizaje con múltiples indicadores, desde una perspectiva escolar, en Galve, J.L. (Coord) (2008). Evaluación e Intervención Psicopedagógica en Contextos Educativos. Vol. I: Estudio de casos de dificultades de lenguaje (oral y lecto-escrito). Vol. II: Problemática asociada con dificultades de aprendizaje. Madrid: EOS.

Vallés Arándiga, A. (2006). Alumnos con inatención, impulsividad e hiperactividad. Intervención multimodal. Colección Fundamentos psicopedagógicos. Madrid: EOS.

Vallés-Arándiga, A. (2008). Programa para el tratamiento educativo de la hiperactividad. ATRE- 
SO-1: Atención, Reflexión y Sosiego. Madrid: EOS.

Vallés Arándiga, A. (2008). Programa para el tratamiento educativo de la hiperactividad.
ATRESO-2: Atención, Reflexión y Sosiego. Madrid: EOS.

Weiss, G. y Hechtman, L. (1992). Hyperactive Children Grown Up. New York: Guilford Press.

Manuscrito recibido: 25/09/2009

Revisión recibida: 13/10/2009

Manuscrito aceptado: 18/10/2009 